Case Report

\title{
Anesthetic Overdose Leading to Cardiac Arrest Diagnosed by End-Tidal Inhalant Concentration Analysis in a Dog
}

\author{
Erik Hofmeister \\ Department of Small Animal Medicine and Surgery, College of Veterinary Medicine, University of Georgia, Athens, GA 30602, USA
}

Correspondence should be addressed to Erik Hofmeister; kaastel@gmail.com

Received 25 March 2013; Accepted 19 May 2013

Academic Editors: C. Gutierrez and M. Woldemeskel

Copyright (C) 2013 Erik Hofmeister. This is an open access article distributed under the Creative Commons Attribution License, which permits unrestricted use, distribution, and reproduction in any medium, provided the original work is properly cited.

\begin{abstract}
A 5-year-old male-castrated Cocker Spaniel presented to the Veterinary Teaching Hospital of the University of Georgia for a total ear canal ablation. Premedication was with carprofen $2.2 \mathrm{mg} / \mathrm{kg} \mathrm{SQ}$, hydromorphone $0.1 \mathrm{mg} / \mathrm{kg} \mathrm{IM}, \mathrm{diazepam} 0.2 \mathrm{mg} / \mathrm{kg}$ IM, and glycopyrrolate $0.01 \mathrm{mg} / \mathrm{kg}$ IM. The patient was induced with lidocaine $2 \mathrm{mg} / \mathrm{kg}$ IV and etomidate $1 \mathrm{mg} / \mathrm{kg}$ IV and maintained with sevoflurane and a constant rate infusion consisting of lidocaine $0.05 \mathrm{mg} / \mathrm{kg} / \mathrm{min}$. Before surgery start, the patient's systolic arterial blood pressure was $110 \mathrm{mmHg}$, heart rate (HR) was 85 beats/min, respiratory rate was 8 breaths/min, end-tidal sevoflurane concentration was $3.2 \%$, and end-tidal $\mathrm{CO}_{2}\left(\mathrm{ETCO}_{2}\right)$ was $23 \mathrm{mmHg}$. As a scrub was being performed, the patient's $\mathrm{HR}$ abruptly dropped to 20 beats/min over the course of 2 minutes. His $\mathrm{ETCO}_{2}$ simultaneously decreased to $16 \mathrm{mmHg}$. At this time, cardiopulmonary arrest was diagnosed. After two minutes of resuscitation, a spontaneous heart beat was obtained and the patient was successfully recovered and discharged without further incident. The cardiac arrest in this case is most likely attributable to an overdose of inhalant anesthesia, which was diagnosed by an anesthetic inhalant concentration monitor. A gas analyzer may be a helpful contribution to the small animal practitioner, particularly those performing more lengthy or complex procedures.
\end{abstract}

\section{Introduction}

Anesthesia in pet dogs can be associated with intraoperative fatality due to the anesthesia. In people, the incidence of anesthetic-related arrest is approximately 1 in 40,000 patients [1]. In small animals, this value has been estimated at 2 in 1,000 [2], which represents a significantly greater risk than in people. In dogs undergoing anesthesia, successful return of spontaneous circulation occurs in approximately $50 \%$ of patients, indicating an anesthetic-related mortality rate of 1 in 1,000 [2]. The incidence of anesthetic arrest in small animals in general practice is unknown, but the incidence of perioperative fatality in dogs has been estimated at 2 in 100, which reflects a marked increase compared to a university setting $[2,3]$. It is possible that this difference is due to increased expertise at an institution, increased vigilance, or greater monitoring technology and interventional capabilities.

A gas analyzer is a device that is equipped to measure endtidal concentrations of various gasses and vapors, notably $\mathrm{CO}_{2}$ and halogenated inhalant anesthetics [4]. A number of technologies can be used to determine this information, with the most simple being infra-red, whereby infra-red light of a specific wavelength is transmitted into a sample, and the amount of light sent out versus the amount of light received by a sensor indicates the concentration of the substance. The American Society of Anesthesiologists requires end-tidal $\mathrm{CO}_{2}\left(\mathrm{ETCO}_{2}\right)$ monitoring for people under anesthesia [5], but this recommendation has not been adopted by the American College of Veterinary Anesthesia. $\mathrm{ETCO}_{2}$ monitoring has been well correlated with outcome during cardiopulmonary resuscitation in small animals [2]. Monitoring end-tidal inhalant anesthetics has been speculated as one possible explanation for an improvement in outcome with anesthetic arrests at a university $[2,6]$.

\section{Case Presentation}

A 5-year-old male-castrated Cocker Spaniel presented to the Veterinary Teaching Hospital of the University of Georgia for a total ear canal ablation (TECA) due to chronic unresolving otitis external and media. Six months earlier, the same procedure had been performed on the left ear without complication. 
On physical exam, the dog was bright, alert, and responsive and had a grade II/VI systolic heart murmur, severe otitis externa, and a normal temperature, pulse, and respiration. There were no significant abnormalities in the history except for the chronic otitis. Bloodwork, including a complete blood count, biochemical profile, and urinalysis revealed a mild neutrophilia with regenerative left shift and a $3+$ proteinuria. The owners declined an echocardiogram.

The patient was premedicated with carprofen $2.2 \mathrm{mg} / \mathrm{kg}$ SQ, hydromorphone $0.1 \mathrm{mg} / \mathrm{kg} \mathrm{IM}$, diazepam $0.2 \mathrm{mg} / \mathrm{kg} \mathrm{IM}$, and glycopyrrolate $0.01 \mathrm{mg} / \mathrm{kg}$ IM. This was the same protocol he had received for his first TECA procedure 6 months before. After 30 minutes, an IV catheter was placed and the patient was induced with lidocaine $2 \mathrm{mg} / \mathrm{kg} \mathrm{IV} \mathrm{and}$ etomidate $1 \mathrm{mg} / \mathrm{kg}$ IV. Etomidate was used to minimize any cardiovascular depression associated with induction, in deference to the heart murmur which had not been fully worked up. The patient was maintained with sevoflurane in oxygen and a constant rate infusion (CRI) consisting of lidocaine $0.05 \mathrm{mg} / \mathrm{kg} / \mathrm{min}$. This was used to provide analgesia and allow for a lower concentration of inhalant anesthesia. The patient was instrumented with indirect blood pressure via doppler, an ECG, a pulse oximeter, and a capnograph/gas analyzer. Throughout the surgical prep, all vitals were stable.

After preparing the patient for surgery, he was moved to the operating room, and intermittent positive-pressure ventilation (IPPV) provided by a mechanical ventilator was begun to deliver 12 breaths a minute with a peak inspiratory pressure of $14 \mathrm{~cm} \mathrm{H}_{2} \mathrm{O}$. At that time, his systolic arterial blood pressure (SAP) was $110 \mathrm{mmHg}$ (normal >90 $\mathrm{mmHg}$ ), heart rate $(\mathrm{HR})$ was 85 beats/min, respiratory rate $(\mathrm{RR})$ was 8 breaths $/ \mathrm{min}$, temperature was $98.2^{\circ} \mathrm{F}$, end-tidal sevoflurane concentration was $3.2 \%, \mathrm{SpO}_{2}$ was $99 \%$, and $\mathrm{ETCO}_{2}$ was $23 \mathrm{mmHg}$ (normal: $35-45 \mathrm{mmHg}$ ). As a surgical scrub was being performed, the patient's $\mathrm{HR}$ abruptly dropped to 20 beats/min over the course of 2 minutes. His $\mathrm{ETCO}_{2}$ simultaneously abruptly decreased to $16 \mathrm{mmHg}$.

At this time, cardiopulmonary arrest (CPA) was diagnosed. The patient's sevoflurane and lidocaine were discontinued, and he was given atropine $0.05 \mathrm{mg} / \mathrm{kg} \mathrm{IV} \mathrm{and}$ epinephrine $0.01 \mathrm{mg} / \mathrm{kg} \mathrm{IV}$, and external chest compressions were initiated. After two minutes of resuscitation, a spontaneous heart beat was obtained. The patient was moved out of the operating room and given mannitol $0.5 \mathrm{~g} / \mathrm{kg} \mathrm{IV}$ over 10 minutes to treat suspected cerebral edema from ischemia during the CPA. Fifty minutes after the CPA event, the patient was extubated. Over the next two hours, the patient regained first his palpebral reflex and then his menace reflex. Surgery was rescheduled for two weeks later, at which time no significant complications were encountered. An echocardiogram was performed prior to this later procedure and mild mitral regurgitation was diagnosed.

\section{Discussion}

The cardiac arrest in this case is most likely attributable to an overdose of inhalant anesthesia. Although the patient did have some underlying cardiac disease, the arterial blood pressure and heart rate were stable throughout surgical prep. No arrhythmias consistent with myocardial disease or ischemia were detected. Also, the patient responded rapidly to treatment with external chest compressions, atropine, and epinephrine. If the cardiac arrest was due to cardiac failure, it would be expected that the patient would have been less responsive to the cardiac resuscitation measures. Furthermore, the patient underwent anesthesia two weeks later without incident and had an echocardiogram which was unremarkable at that time.

Other possible causes include excessive vagal tone, a machine fault (such as a closed pop-off valve), or overdose of a different drug. There was no event surrounding the CPA which may precipitate vagal hypertonus, such as the manipulation of the eye or viscera. No machine fault was detected at the time, nor afterwards when the machine was evaluated thoroughly. No other drugs were being given with the exception of lidocaine. Lidocaine is a negative inotrope and can depress the cardiac output at toxic doses. However, the dose of lidocaine was checked and the syringe pump administering the CRI was also confirmed to be administering a proper concentration.

Anesthetics have a fairly narrow therapeutic index (TI), which is the $\mathrm{LD}_{50} / \mathrm{ED}_{50}$. Sevoflurane has a TI of 2.6 in rats [7] and propofol has a TI of 3.1 in rats [8]. Therefore, overdose with inhalant agents is relatively easy. The $\mathrm{ED}_{50}$ of inhalant anesthetics is the minimum alveolar concentration (MAC), the point at which $50 \%$ of patients will not move in response to a standard painful stimulus. The MAC for sevoflurane is approximately $2.1 \%$ [9]. For a surgical plane of anesthesia, typically $1.2-1.5$ times the MAC is necessary (i.e., 2.5-3.2\% for sevoflurane) [10]. However, this requirement will be decreased by other anesthetic and analgesic drugs. In this patient, the time of CPA was 2 hours after premedication, so it is unlikely that the premedicants were still exerting a significant MAC-sparing effect. However, the patient was concurrently receiving lidocaine.

Lidocaine is a fast sodium channel blocker which has potent analgesia and sedative properties in dogs. It profoundly decreases the MAC requirement in dogs, by up to $23 \%$ at the dose given to this patient [11]. Therefore, it would be expected that the patient could have been maintained at an end-tidal sevoflurane concentration between 1.9 and $2.4 \%$. An end-tidal concentration of $3.2 \%$, which was the concentration at the time of CPA, represents a 33\% higher concentration than the highest which would be necessary to keep the patient adequately anesthetized for surgery. At the time of CPA there was no surgical stimulation. Typically, surgical stimulation partially counteracts the cardiodepressant effects of the inhalant anesthetics. Thus, this patient was at a higher concentration of sevoflurane than that required for surgery, was not actively receiving surgical stimulation, and was on a lidocaine CRI which may have been blunting any cardiovascular stimulus he had. This particular synergy of events likely led to the CPA event.

This case was unusual because the cause of CPA was very likely inhalant anesthesia overdose, and the concentration of inhalant at the time of CPA was known. Although endtidal gas analyzers are expensive (ranging in price from $\$ 2,800$ to $\$ 15,000$ ), they are useful for documenting the 
concentration of inhalant in the patient which helps guide clinical decision making and is the only way to definitively diagnose inhalant anesthesia overdose or underdose. There has been speculation that the presence of a gas analyzer dramatically improves the ability to manage equine cases [6], and this may also be true in small animals. A gas analyzer may therefore be a helpful contribution to the small animal practitioner, particularly those performing more lengthy or complex procedures. This case also highlights the potential risk with administering drugs as a CRI which significantly decreases the MAC, such as lidocaine, morphine, and/or ketamine [12]. Although balanced anesthesia with a variety of drugs each acting to reduce the dose of any one drug is generally preferable, in inexperienced hands these techniques may be dangerous. The clinician should always be thoroughly comfortable with the anesthesia technique employed and understand the effects of drugs on the inhalant anesthetic requirement.

\section{References}

[1] C. C. P. Eagle and N. J. Davis, "Report of the anaesthetic mortality committee of Western Australia 1990-1995," Anaesthesia and Intensive Care, vol. 25, no. 1, pp. 51-59, 1997.

[2] E. H. Hofmeister, B. M. Brainard, C. M. Egger, and S. Kang, "Prognostic indicators for dogs and cats with cardiopulmonary arrest treated by cardiopulmonary cerebral resuscitation at a university teaching hospital," Journal of the American Veterinary Medical Association, vol. 235, no. 1, pp. 50-57, 2009.

[3] D. C. Brodbelt, K. J. Blissitt, R. A. Hammond et al., "The risk of death: the confidential enquiry into perioperative small animal fatalities," Veterinary Anaesthesia and Analgesia, vol. 35, no. 5, pp. 365-373, 2008.

[4] J. A. Dorsch and S. E. Dorsch, "Gas monitoring," in Understanding Anesthesia Equipment, J. A. Dorsch and S. E. Dorsch, Eds., pp. 679-750, Williams \& Wilkins, Philadelphia, Pa, USA, 4th edition, 1999.

[5] J. Weaver, "The latest ASA mandate: $\mathrm{CO}_{2}$ monitoring for moderate and deep sedation," Anesthesia Progress, vol. 58, no. 3, pp. 111-112, 2011.

[6] A. K. J. Parviainen and C. M. Trim, "Complications associated with anaesthesia for ocular surgery: a retrospective study 19891996," Equine Veterinary Journal, vol. 32, no. 6, pp. 555-559, 2000.

[7] J. P. Fast, M. G. Perkins, R. A. Pearce, and S. Mecozzi, "Fluoropolymer-based emulsions for the intravenous delivery of sevoflurane," Anesthesiology, vol. 109, no. 4, pp. 651-656, 2008.

[8] J.-X. Zhou, N.-F. Luo, X.-M. Liang, and J. Liu, "The efficacy and safety of intravenous emulsified isoflurane in rats," Anesthesia and Analgesia, vol. 102, no. 1, pp. 129-134, 2006.

[9] E. H. Hofmeister, B. M. Brainard, L. M. Sams, D. A. Allman, and A. M. Cruse, "Evaluation of induction characteristics and hypnotic potency of isoflurane and sevoflurane in healthy dogs," American Journal of Veterinary Research, vol. 69, no. 4, pp. 451456, 2008.

[10] R. H. de Jong and E. I. Eger II, "MAC expanded. $A_{50}$ and $\mathrm{AD}_{50}$ values of common inhalation anesthetics in man," Anesthesiology, vol. 42, no. 4, pp. 384-389, 1975.

[11] J. Wilson, T. J. Doherty, C. M. Egger, A. Fidler, S. Cox, and B. Rohrbach, "Effects of intravenous lidocaine, ketamine, and the combination on the minimum alveolar concentration of sevoflurane in dogs," Veterinary Anaesthesia and Analgesia, vol. 35, no. 4, pp. 289-296, 2008.

[12] W. W. Muir III, A. J. Wiese, and P. A. March, "Effects of morphine, lidocaine, ketamine, and morphine-lidocaine-ketamine drug combination on minimum alveolar concentration in dogs anesthetized with isoflurane," American Journal of Veterinary Research, vol. 64, no. 9, pp. 1155-1160, 2003. 

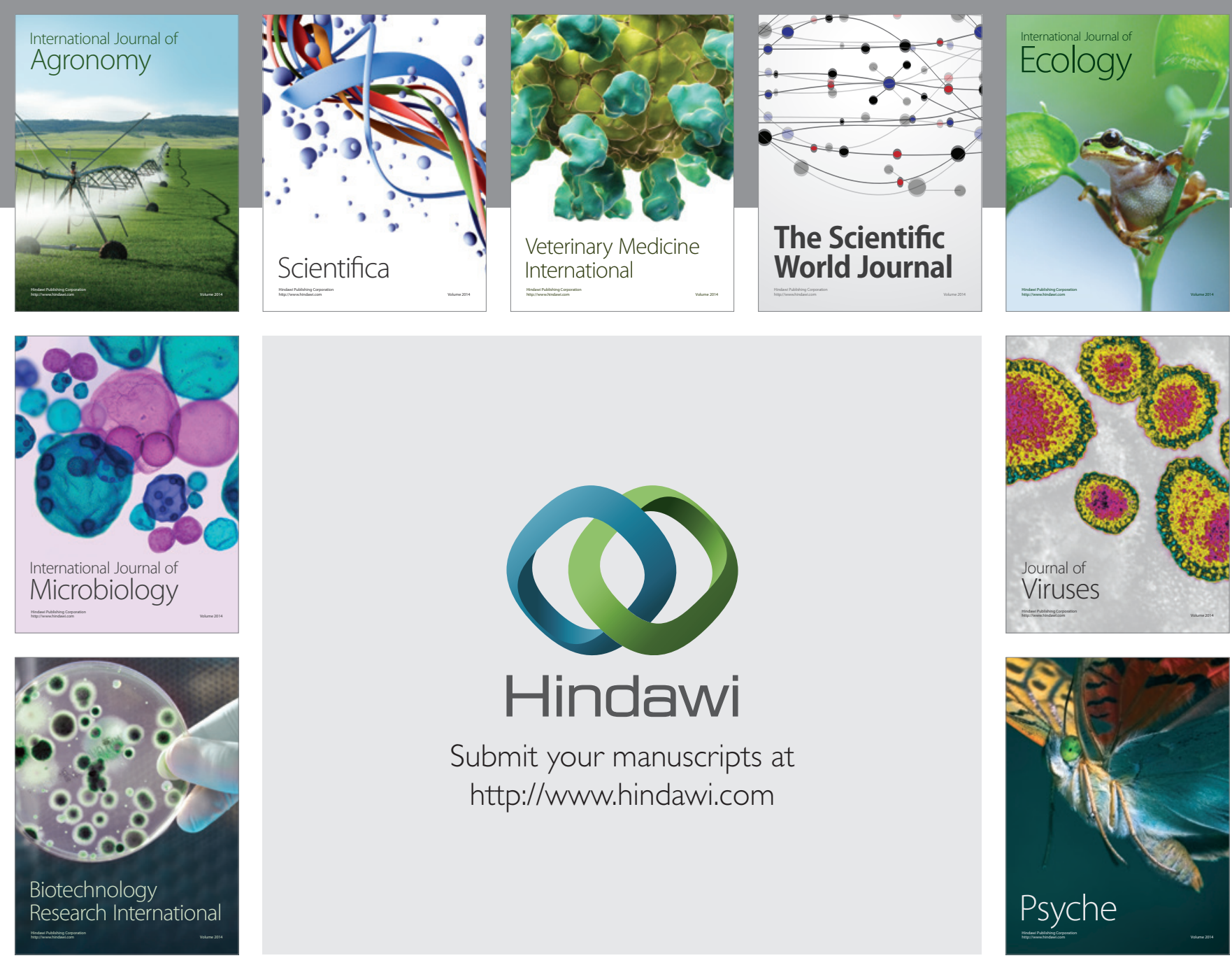

Submit your manuscripts at http://www.hindawi.com
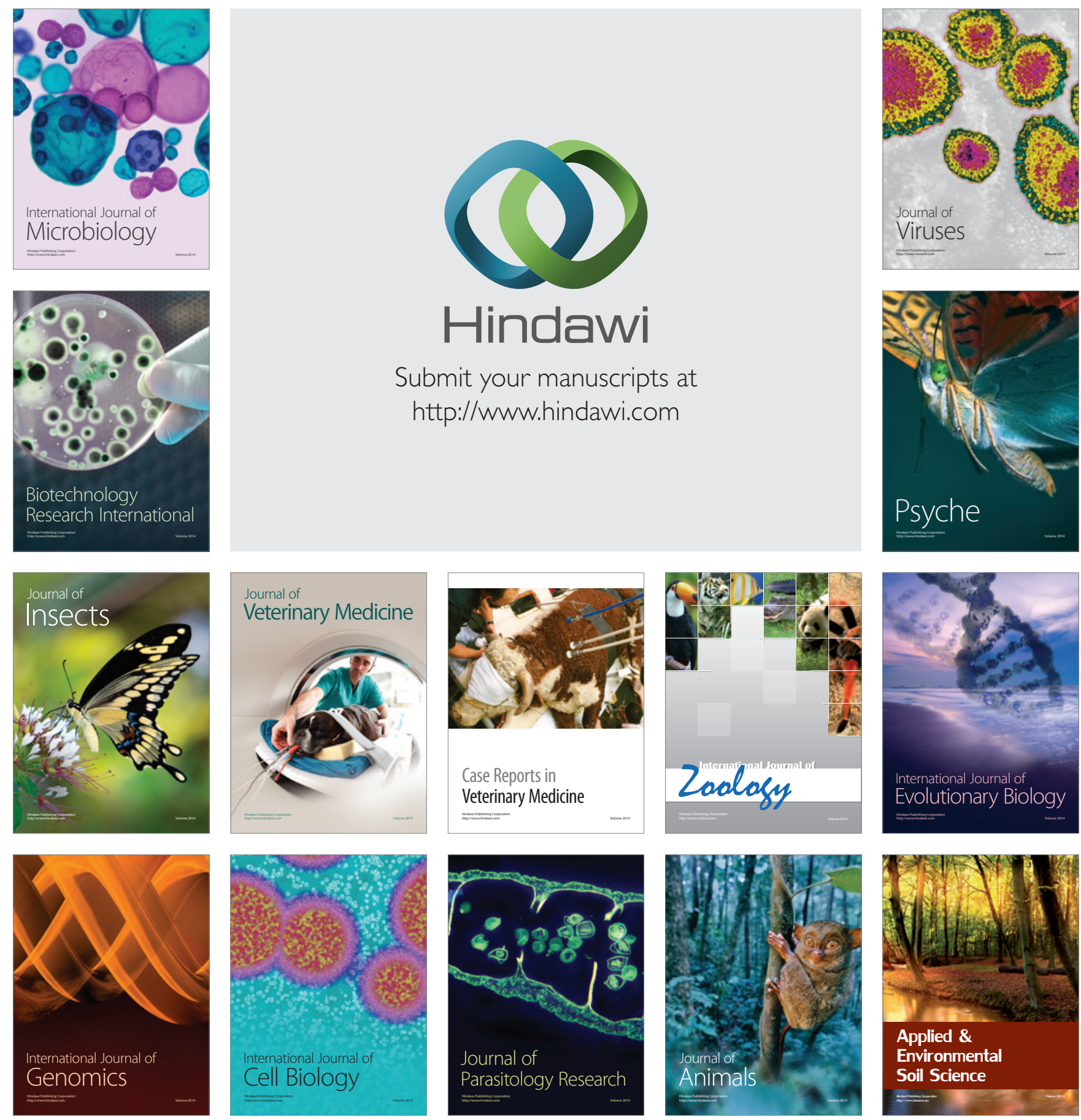